\title{
ELECTRON BEAM SIZE MEASUREMENTS IN A COOLING SOLENOID
}

\author{
T.K. Kroc ${ }^{\#}$, A.V. Burov, T.B. Bolshakov, A. Shemyakin, \\ FNAL*, Batavia, IL 60510, USA, S.M. Seletskiy, University of Rochester, USA
}

\begin{abstract}
The Fermilab Electron Cooling Project [1] requires a straight trajectory and constant beam size to provide effective cooling of the antiprotons in the Recycler. A measurement system was developed using movable appertures and steering bumps to measure the beam size in a $20 \mathrm{~m}$ long, nearly continuous, solenoid. This paper discusses the required beam parameters, the implimentation of the measurement system and results for our application.
\end{abstract}

\section{INTRODUCTION}

Electron cooling uses a DC beam (up to .5A) of cold electrons moving along a trajectory coincident with the trajectory of a beam of antiprotons to be cooled. Matching the velocities of the two beams allows Coulomb interactions to transfer energy ("temperature") from the antiprotons to the electrons. In addition to the constraint on longitudinal velocities, there are limits on both the beam's inherent transverse temperature and its effective temperature due to trajectory perturbations and remnant variations of its envelope due to the optics of the beam line.

For electron cooling to be effective, the electrons need to have a total transverse motion of no more than $200 \mu \mathrm{rads}[2]$. Of this, a maximum of $100 \mu \mathrm{rads}$ is allocated for variations of the beam envelope. This paper describes a procedure that has been developed to measure the outer envelope of the beam along its trajectory through the cooling section.

The cooling section consists of 10 two meter long solenoids [3]. Before and after the cooling section and between each solenoid is a movable circular aperture, a "scraper", which consists of a copper bar, .125" thick with a $15 \mathrm{~mm}$ hole through which the beam can pass. There are a total of 11 scrapers.

The concept is to insert each scraper so that the DC beam passes through the aperture and then move the beam within the scraper to determine the shape of the beam at that location. This can be done at 11 locations throughout the cooling section to determine the evolution of the beam envelope as a function of the longitudinal position. While we are able to work with pulsed beam, and do so for initial measurements, some phenomenon such as ions may perturb the focusing sufficiently to affect the optics. The beam has very distinct edges which can be used advantageously for determining its size.

*Operated by Universities Research Association Inc. under Contract No. DE-AC0276CH03000 with the United States Department of Energy.

"kroc@fnal.gov
The procedure was tested in a prototype experiment which ended in May of 2004. It was successfully used to decrease the envelope variations to less than $.1 \mathrm{~mm}$ along the length of the cooling section for a $3.5 \mathrm{MeV}$, $350 \mathrm{~mA}$ beam.

\section{TECHNIQUE}

\section{Overview}

The first step of the program is to measure the transfer function of the steering correctors in the beam line. A program is used to systematically increment the current in each corrector and measure the response in every beam position monitor (BPM) downstream.

Another program calculates sets of coefficients (which we call "mults") to use in combinations of correctors that can be varied in unison to move the beam in a systematic way. These movements include a parallel shift and four-bumps. The parallel shift uses two sets of correctors to offset the beam trajectory parallel to its nominal trajectory without concern for restoring the path at any point downstream. This was mainly used during program development. The fourbump uses two additional sets of correctors to restore the original trajectory at some point farther downstream (see figure 1). The corrector sets typically consist of a single corrector but the program can combine them into groups with variable weighting.

A third program uses selected mults to move the beam in the cooling section to explore the aperture of the scrapers. The beam is moved in one of eight selected directions until the edge of the beam encounters the edge of the scraper. The position is noted and the process is repeated until all eight directions have been processed. The program then computes the envelope properties of the beam.

\section{Details}

The scrapers are inserted individually so that the aperture of the scraper is centered on the beam trajectory (Figure 1). A bump is constructed using steering dipoles before and after the cooling section to displace the beam transversely but keep it parallel to the optimal trajectory through the entire cooling section. With one of the scrapers inserted, the beam is turned on. The beam is then moved up/down, left/right, and in $45^{\circ}$ diagonals until it touches the aperture of the scraper. The touch is determined by: the response of a nearby loss monitor, a reduction in intensity in a BPM immediately downstream of the scraper, or current loss. This can be performed with either DC beam, using the current loss or loss monitors, or a pulsed beam, which typically has a pulse length of $2 \mu \mathrm{sec}$ and a repetition rate of $1 \mathrm{~Hz}$, using the BPM response. 


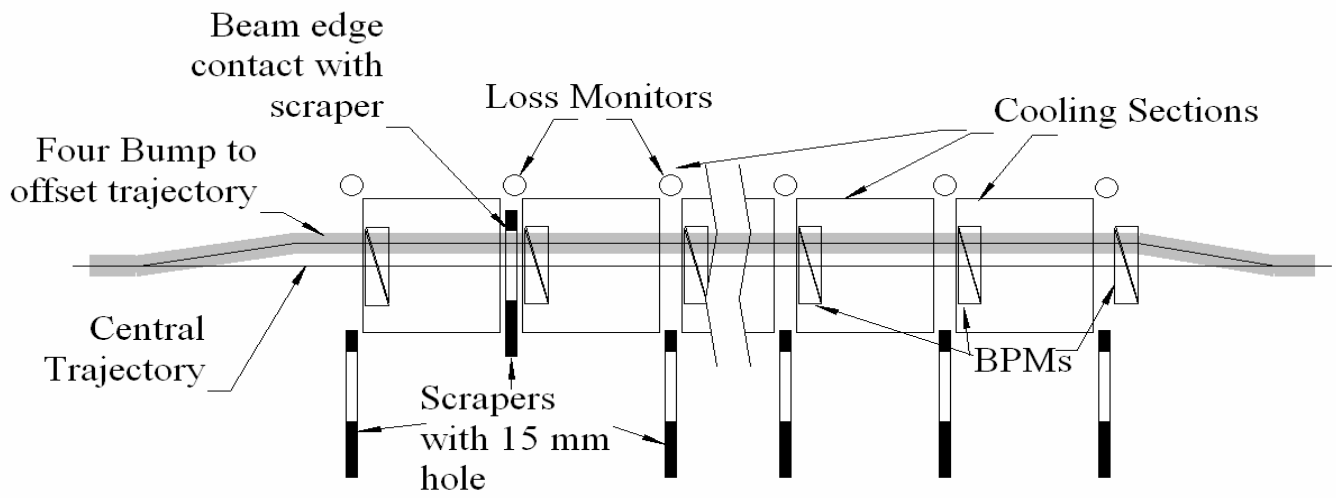

Figure 1 Diagram of the 4-bump through the cooling section.

The eight points of contact provide the information for determining the parameters of the beam envelope. These parameters include: the axes of the ellipse, its eccentricity, and tilt. The eleven ellipses along the length of the cooling section show the evolution of the beam envelope. This information about the variations of the envelope along the length of the solenoid can then be used to adjust the focusing (and trajectory if necessary) into the cooling section to reduce these variations below the allowed tolerances using a procedure described in reference [4].

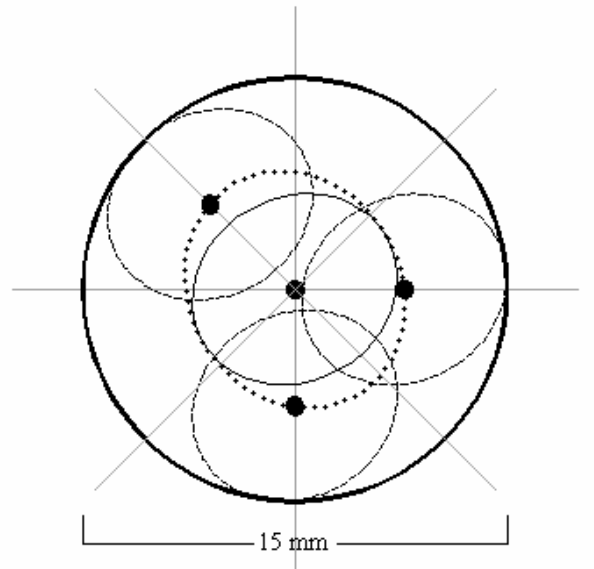

Figure 2 Diagram of the beam envelope as it is scanned around the aperture of the scraper hole. Only three of the eight displaced ellipses are shown for clarity.

Figure 2 illustrates the ellipse finding technique. The bold outer circle is the $15 \mathrm{~mm}$ diameter hole in the scraper. The solid ellipse in the center represents the beam with an area equal to a $7 \mathrm{~mm}$ diameter circle but with an eccentricity of .5 and its semi-major axis rotated counter-clockwise by $30^{\circ}$. Its center is the black dot in the center. The dashed ellipses are the position of the beam when it touches the hole after it has been transposed along one of the directions of movement. The centroids of these ellipses are then used to determine the envelope of the beam. They are fit to an ellipse (the dotted line connecting them). Using the properties of the ellipse, the beam line can be tuned to degenerate the ellipse into a circle. This procedure requires that the largest radius of the envelope be less than the radius of the aperture.

The BPMs have a resolution of $5 \mu \mathrm{m}$. The distance between the scrapers and its BPM is less than $20 \mathrm{~cm}$ while the distance between measurement locations is 2 meters. The beam's Larmor radius is 1 meter. The alignment tolerance of the scrapers is $50 \mu \mathrm{m}$. The limiting uncertainty is therefore the determination of the beam edge. Figure 3 shows a comparison of using loss monitors and measured current losses to determine the beam edge. One can see that the edge of the electron beam is very sharp and can be determined to about $.1 \mathrm{~mm}$. Together these tolerances indicate we should be able to determine the motion of the beam to $\sim 30$ rads.

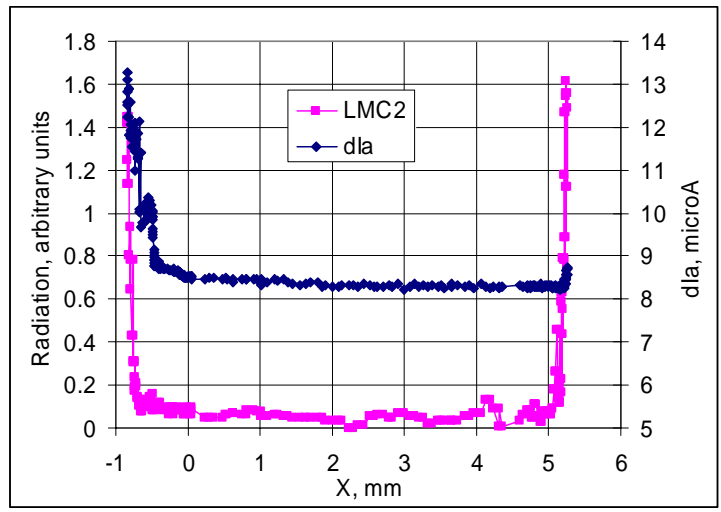

Figure 3 Comparison of determining the beam edge by using loss monitors (LMC2) and measured current losses (dIa). Beam current was $220 \mathrm{~mA}$ and energy 3.5 $\mathrm{MeV}$ during operation of the prototype system.

\section{RESULTS}

Figure 4 shows beam edge measurements for three scrapers that were made during the prototype run of the Electron Cooling system. The scraper ID indicates its position in meters along the cooling section. The beam 
is shown to be mildly eccentric but maintains a relatively uniform shape through the cooling section. During other work in that prototype run, we were able to reduce the beam size variations to $0.4 \mathrm{~mm}$ with a DC beam current of $100 \mathrm{~mA}$. This gave and r.m.s angle of 100 rrads. While a number of issues need to be resolved (see next section), these results indicate that the technique is valid.

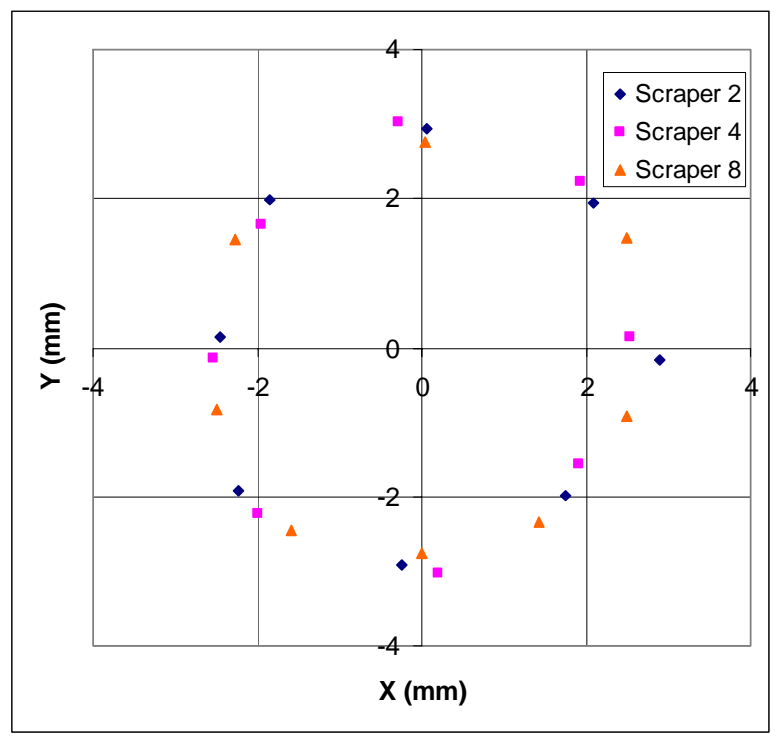

Figure 4 Beam edges for three scrapers in prototype measurements. Beam energy was $3.5 \mathrm{MeV}$. Current was $350 \mathrm{~mA}$.

\section{PRACTICAL CONSIDERATIONS}

A number of considerations come into play when trying to make the program automatic and robust.

The range of the correction elements is not infinite. The program has to make its adjustments in the corrector settings that remain within the range of the power supply. The size of the adjustments must also keep the beam within the aperture of the beam line. This also requires that the nominal trajectory consist of settings that are near the middle of the setting range. The physical limitations of the layout of the beam line do not always allow the placement of correctors and BPMs at their optimum locations. Therefore, some correctors are weak and the coefficients of their mults are large. This results in those correctors ranging out quickly and limits the physical range of the scanning.

A large portion of the electron cooling beam line shares a tunnel enclosure with the Fermilab Main Injector (MI). Because we have not yet fully magnetically shielded the beam line, beam motion due the ramp of the MI causes beam motion in some areas of the line. Therefore position measurements must be synced to the repetition rate of the MI in order to make the measurements at quiet moments in the MI cycle.
This slows the process as it reduces the frequency at which measurements can be made.

The high beam power (over 2MW instantaneous) requires special considerations. Direct strikes of the beam on beam line components can be very damaging. Interruption of the recirculation of the electron beam can lead to discharges in the Pelletron electron source. Therefore, during development, we typically used a pulsed beam. The amplitude of the beam can vary up to the full current $(.5 \mathrm{~A})$ and typically has a pulse length of $2 \mu \mathrm{sec}$ and a repetition rate of $1 \mathrm{~Hz}$.

The different running modes (pulsed or DC) affect how beam contact with the scrapers is determined. When using DC beam, loss monitors can be used to detect the radiation generated by the electrons striking the scrapers. Thresholds for determining contact can be set rather high, many times background readings. When running with pulsed beam, especially at lower beam currents, the radiation produced is below the detection threshold of the loss monitors. In this case we rely on the intensity of the signal from the BPMs. If beam strikes the BPM plates then the readings become unreliable and are useless for determining contact. Therefore, the detection threshold has to be quite tight, deviations of no more then $2 \%$.

The proximity to the MI also interferes with the determination of contact with the scraper edges. Beam loss in the MI at various points in its cycle can interfere with the loss monitor signal. Again the remedy is to sync the readings with the MI repetition rate.

\section{CONCLUSIONS}

A technique has been developed for measuring the size, shape, and uniformity of a DC electron beam through the Fermilab Electron Cooling cooling section. Measurements made with a prototype layout indicate that the technique will be able to provide the data necessary to tune the optics of the line to keep the transverse velocity parameters of the beam within our tolerances. Experimental results have already shown r.m.s. angles of $100 \mu \mathrm{rads}$ while tolerances in the final installation predict $30 \mu \mathrm{rads}$.

\section{REFERENCES}

[1] S. Nataitsev, et. al., "Commissioning of Fermilab's Electron Cooling System for 8-GeV Antiprotons", these procedings.

[2] A. Burov, "Electron Cooling Scenarios at Fermilab", NIM-A 532(2004) p. 291-297.

[3] Leibfritz, et. al., "Fermilab Electron Cooling Project : Engineering Aspects of Cooling Section", Proc. 2001 Part. Acc. Conf., pg. 1414.

[4] A. Burov and V. Lebedev, "Cylindric Electron Envelope for Relativistic Electron Cooling", FERMILAB-TM-2303-AD (2005). 